\title{
La représentation des guerres et de l'histoire américaine de la seconde moitié du XXe siècle dans Code Quantum (Quantum Leap, NBC, 1989-1993)
}

\section{Claire Cornillon}

\author{
(2) OpenEdition \\ Journals \\ Édition électronique \\ URL : http://journals.openedition.org/tvseries/1937 \\ DOI : 10.4000/tvseries.1937 \\ ISSN : 2266-0909 \\ Éditeur \\ GRIC - Groupe de recherche Identités et Cultures
}

Référence électronique

Claire Cornillon, « La représentation des guerres et de l'histoire américaine de la seconde moitié du XXe siècle dans Code Quantum (Quantum Leap, NBC, 1989-1993) », TV/Series [En ligne], 10 | 2016, mis en ligne le 01 décembre 2016, consulté le 01 mai 2019. URL : http://journals.openedition.org/ tvseries/1937 ; DOI : 10.4000/tvseries. 1937

Ce document a été généré automatiquement le 1 mai 2019.

\section{(c) (i) (9)}

TV/Series est mis à disposition selon les termes de la licence Creative Commons Attribution - Pas d'Utilisation Commerciale - Pas de Modification 4.0 International. 


\title{
La représentation des guerres et de l'histoire américaine de la seconde moitié du XXe siècle dans Code Quantum (Quantum Leap, NBC, 1989-1993)
}

\author{
Claire Cornillon
}

Quantum Leap ${ }^{1}$ (Code Quantum en français), série américaine créée par Donald Bellisario, producteur important de la télévision américaine à qui l'on doit notamment Magnum (CBS, 1980-1988), est diffusée aux États-Unis de 1989 à 1993 (à partir de 1993 en France sur Série Club et M6). Elle met en scène un scientifique, le Dr Samuel Beckett, qui voyage dans le temps en endossant temporairement l'identité et l'apparence d'une personne pour changer son destin et « réparer les erreurs du passé ${ }^{2}$ ». Elle compte cinq saisons de vingtdeux épisodes d'une quarantaine de minutes ${ }^{3}$. La série a obtenu une reconnaissance critique et des prix et a suscité l'enthousiasme d'un public fidèle, notamment grâce au duo constitué par Scott Bakula qui incarne Sam Beckett (Golden Globe du meilleur acteur en 1992) et Dean Stockwell, interprétant son meilleur ami, l'amiral Al Calavicci (Golden globe du meilleur acteur dans un second rôle en 1990). Les deux personnages se complètent et n'ont pas la même vision de la vie: Al est un militaire, aux options idéologiques parfois conservatrices, mais qui est aussi un homme à femmes, alors que Sam est l'incarnation de l'homme vertueux qui défend tous les opprimés et dont les opinions sont souvent plus progressistes. Au-delà de ce duo, c'est aussi pour son concept et sa structure, qui fait que chacun des épisodes explore un moment de l'histoire américaine contemporaine, que la série a marqué, offrant un certain nombre de scènes mémorables où Sam rencontre des figures historiques ou des célébrités, comme Michael Jackson, Stephen King ou Woody Allen. 
Figure 1: Sam Beckett (Scott Bakula) et Al Calavicci (Dean Stockwell) dans l'épisode pilote

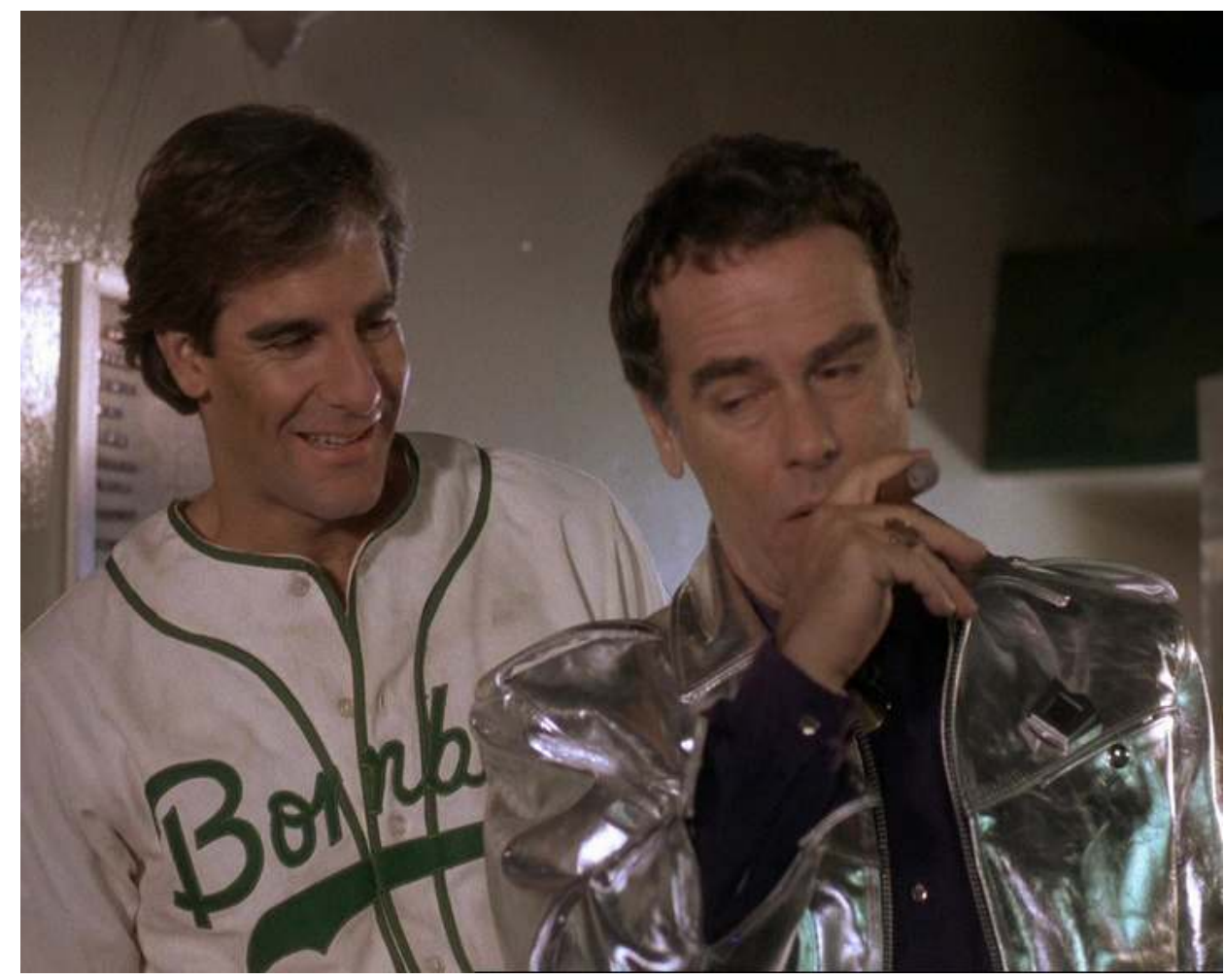

Le Dr Beckett ne peut voyager que dans le temps de sa propre vie, ce qui implique que les épisodes prennent place, en principe, entre 1953 et $1985^{4}$. La série propose donc une fresque touchant à trois décennies de l'histoire des États-Unis et, par là-même, est hantée par les différents conflits de la seconde moitié du XXe siècle, notamment les conséquences de la Seconde Guerre mondiale et de la guerre de Corée, ainsi que la guerre froide et la guerre du Vietnam. Sur l'ensemble des cinq saisons, dix-huit épisodes abordent de manière significative ces guerres : neuf portent sur le Vietnam, quatre sur la Seconde Guerre mondiale, quatre sur la guerre froide, un sur la guerre de Corée, et un sur la guerre de Sécession ${ }^{5}$. Il s'agit donc là de l'un des thèmes les plus fréquemment traité par la série, avec celui de la lutte pour les droits civiques et celui de l'évolution de la condition des femmes, autant de problématiques socio-politiques structurantes de l'histoire des États-Unis, en particulier dans la seconde moitié du XXe siècle.

En effet, les guerres dans Quantum Leap ne constituent pas seulement un décor. Au contraire, elles sont au cœur des intrigues de bien des épisodes et touchent à l'enjeu même de la série : aborder sans cesse les moments majeurs de l'histoire des États-Unis par le biais de la petite histoire des individus. Sam Beckett tente d'éviter des drames mais suscite également des destins qui vont, chacun à leur échelle, jouer un rôle au sein de la collectivité. Les changements dont il est à l'origine sont localisés mais significatifs pour les individus concernés. Il ne s'agit pas, pour Sam Beckett, de modifier l'Histoire avec un grand $\mathrm{H}$ mais d'avoir un impact sur l'existence de certains individus et d'expérimenter les événements par le regard des personnes à qui il emprunte temporairement la vie. Dès lors, c'est lui-même qui change également, tout comme Al qui le suit dans ses voyages.

4 La série applique cette approche, en partie didactique, à la représentation de la guerre, en construisant un propos pacifiste à travers un travail sur le point de vue. Ainsi, bien que 
l'on connaisse l'attrait de Donald P. Bellisario (le créateur de la série) pour les thématiques militaires ${ }^{6}$, Quantum Leap développe cependant cette dimension pacifiste qui, si elle n'est pas radicale, vient remettre en question un certain nombre de conflits en insistant sur la souffrance qu'ils ont causée. L'ensemble de la série est une manière de mettre en perspective la société américaine contemporaine en remontant à divers événements qui l'ont construite, et notamment les différents conflits dans lesquels les États-Unis se sont impliqués au XXe siècle et qui se poursuivent au moment de la diffusion de la série elle-même, puisqu'elle est contemporaine de la guerre du Golfe.

\section{Modalités de représentation de la guerre dans Quantum Leap}

5 La guerre est présente selon trois modalités principales dans la série : la représentation du champ de bataille, le récit -lorsque l'expérience de la guerre est racontée par un personnage dans le cadre d'un dialogue - et ce que l'on pourrait appeler la trace, c'est-àdire les images et motifs telles que les blessures ou les uniformes qui évoquent la guerre sans la montrer directement. La première - la représentation directe - est peu utilisée dans la série mais l'usage récurrent des récits et des traces des guerres va souligner l'omniprésence de la guerre dans la société et dans la vie des individus, comme un contexte sous-jacent qui explique des itinéraires de vie brisées, alors même que le combat n'est pas sur le territoire américain. Ce dispositif s'appuie sur l'idée que le hors champ se manifeste constamment dans le champ de manière plus ou moins indirecte. En effet, puisque chaque épisode se déroule dans un contexte spatio-temporel et avec des personnages différents, chaque motif qui revient ressort nécessairement de manière plus visible au sein de cette diversité. Le nombre et la variété des personnages concernés par la guerre dans la série souligne la manière dont elle affecte l'intégralité de la société, quels que soient les époques, les lieux, l'âge, le genre ou le milieu social des individus.

\subsection{Une représentation iconique de la guerre et de l'Histoire}

6 La série se déroule presque exclusivement sur le territoire américain. De toute évidence, la série est limitée par ses restrictions budgétaires : il est clair que la production d'un film d'époque avec un casting entièrement renouvelé chaque semaine constitue une gageure pour une série de la fin des années 1980 et l'on sait que les producteurs de la série ont rencontré des difficultés financières croissantes jusqu'à l'arrêt final de la série. Trois épisodes seulement se passent hors des États-Unis dans l'ensemble de la série et il s'agit d'épisodes très particuliers : l'épisode portant sur un faux vampire qui se passe à Londres (5.15) et le double épisode qui ouvre la saison 5, centré sur Lee Harvey Oswald. Certaines scènes s'y passent au Japon ou en URSS.

C'est aussi l'une des seules fois où la série touche de près à la grande Histoire puisque Sam croit devoir sauver le président Kennedy. Il échoue finalement et sauve Jackie Kennedy qui serait également morte sans son intervention. Le seul autre exemple est le deuxième épisode de la saison 3, «The Leap Home, Part II », où Sam se retrouve auprès de son frère Tom à Saigon pendant la Guerre du Vietnam. Cependant, en dehors de ces considérations contextuelles, les limites spatio-temporelles de la série ajoutent aussi à l'identité forte de sa formule et créent des effets de lecture indéniables en termes idéologiques. De fait, la clôture sur le territoire des États-Unis implique une concentration sur le point de vue 
américain, à l'exclusion des lectures étrangères de ces conflits. Quelques exceptions sont néanmoins à noter, comme l'épisode centré sur l'épouse japonaise d'un ancien soldat, « The Americanization of Machiko » (2.3).

Figure 2 : Sam Beckett en soldat lors de la Guerre du Vietnam (3.2)

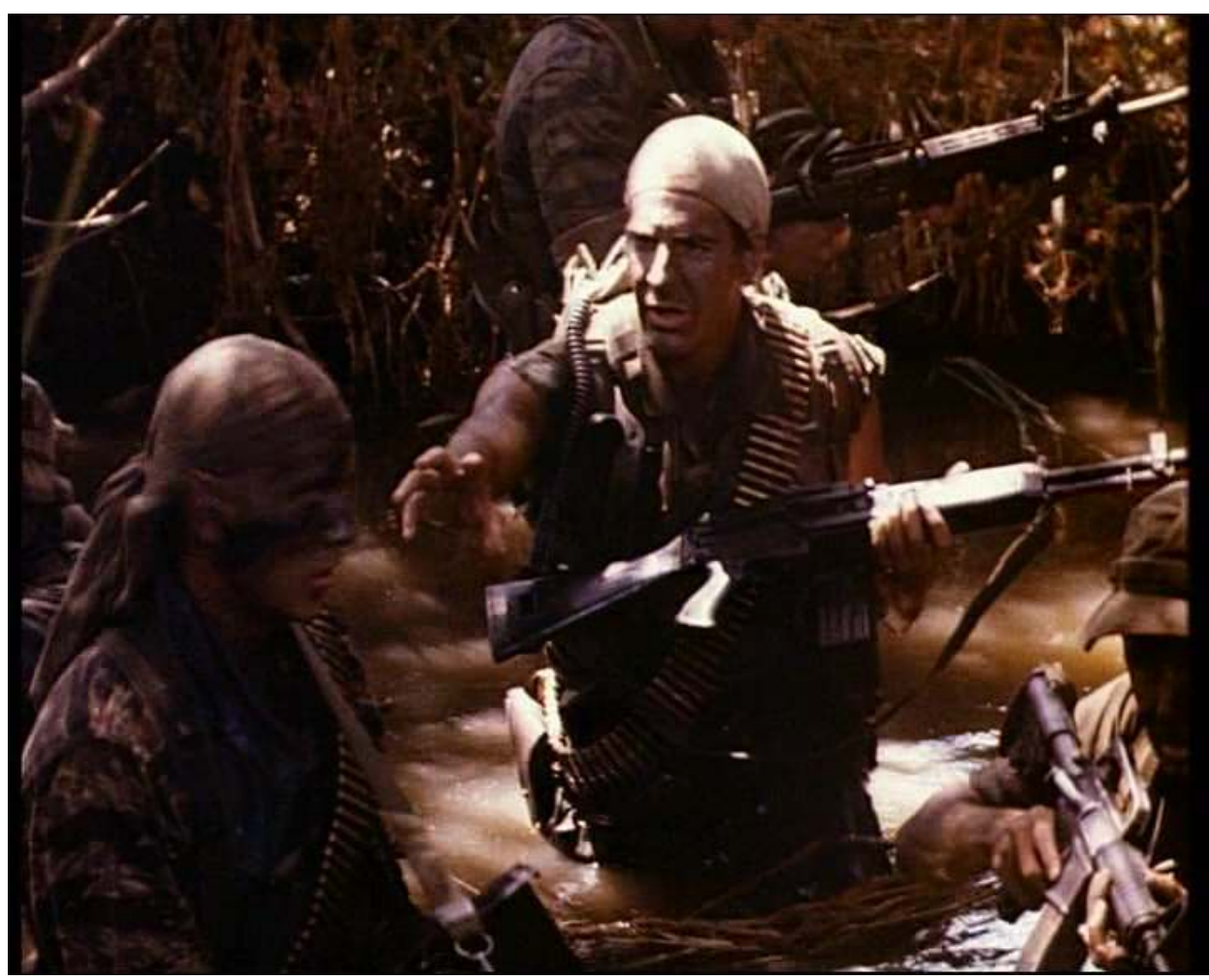

Dans « The Leap Home, Part II », qui constitue l'une des seules représentations directes du champ de bataille dans la série ${ }^{7}$, on retrouve une imagerie classique de la guerre du Vietnam qui reprend l'ensemble des motifs des films les plus connus sur ce conflit: les hélicoptères (comme dans Apocalypse Now, Francis Ford Coppola, 1979), la jungle (comme dans Platoon, Oliver Stone, 1986), les prisonniers (comme dans The Deer Hunter, Michael Cimino, 1978). On touche à une caractéristique importante de Quantum Leap qui est sa dimension iconique: Sam se promène dans l'histoire mais il se promène aussi dans les écrans, dans l'imagerie américaine de la seconde moitié du XXe siècle, dans la mémoire visuelle du téléspectateur. Ainsi le générique fait alterner des photographies d'époque, devenues célèbres - telles qu'un portrait de Jimi Hendrix ou un autre de John Fitzgerald Kennedy, une image de Neil Armstrong sur la lune - et des images fictionnelles de Sam issues de la série.

\subsection{Une mémoire imprégnée par la culture populaire}

Sam est dès lors inséré dans l'histoire mais aussi dans la mémoire visuelle de l'Amérique et du spectateur. Non seulement il vit la vie d'une personne différente chaque semaine, mais il incarne aussi un personnage différent à chaque épisode, comme un comédien. Cette mise en abyme s'appuie notamment sur un imaginaire cinématographique très présent dans la série, ainsi que sur des références télévisuelles, puisque Sam regarde souvent la télévision, et en particulier les émissions de son enfance. La mémoire télévisuelle et cinématographique est fondamentale dans la série. Quantum Leap se 
réinvente chaque semaine, notamment par la référence générique : chaque épisode porte sur un sujet différent, possède une ambiance spécifique et revisite souvent un motif ou un genre de l'histoire du cinéma ou de la télévision, tels que le polar ou le film d'horreur. Certains épisodes se fondent d'ailleurs sur des références directes à des films («Miss Melny et son chauffeur " (The Color of Truth, 1.7) est une référence à Miss Daisy et son chauffeur (Driving Miss Daisy, Bruce Beresford, 1989); l'épisode final de la saison 1 (« Play it again, Seymour " 1.9) rend hommage aux films avec Humphrey Bogart et évoque la piècehommage de Woody Allen, Play it again, Sam (1969).

Dans le cas de l'épisode 3.2 sur la guerre du Vietnam, la série propose une référence au genre du film de guerre. Il ne s'agit pas seulement de pastiche, car ce qui va faire l'intérêt de l'épisode est la manière dont s'articulent ces motifs et ces références avec la mythologie et la structure propres à la série. Ce qui change du film de guerre classique, dans cet épisode, est que le personnage connaît l'avenir et essaie de sauver son frère d'une mort prématurée. L'épisode est centré sur le problème même de la modification d'événements du passé, puisqu'en sauvant son frère, Sam cause la mort d'une journaliste. Par ailleurs, l'épisode soulève la question de la différence entre les objectifs personnels de Sam et sa mission puisque celui-ci se rend compte finalement que c'est à cette époque qu'Al a été fait prisonnier et qu'il aurait pu le sauver si Al l'avait prévenu. Mais l'amiral a privilégié la mission sur son propre destin. Dans le contexte de cette reprise du film de guerre, les enjeux dramatiques sont pourtant tout à fait spécifiques à la série elle-même.

L'ensemble de la série, et notamment sa fin, se fonde sur l'idée de sacrifice du héros qui renonce à sa vie personnelle pour œuvrer pour le bien des autres. La décision d'Al dans cet épisode fait donc écho à l'itinéraire éthique suivi par Sam. Le contexte guerrier, redoublé par l'implication personnelle des personnages dans l'histoire de cet épisode, cristallise les tensions narratives qui structurent la série. La construction sérielle de Quantum Leap repose donc sur la réitération d'un même système de valeurs tout au long des épisodes. Dans chacun d'eux, les personnages spécifiques à l'histoire de la semaine, mais aussi les deux protagonistes, font face à des choix éthiques et les valeurs qui sont prônées sont toujours identiques. C'est en cela que se construit la dynamique didactique de la série. 
Figure 3 : Al prisonnier au Vietnam (3.2)

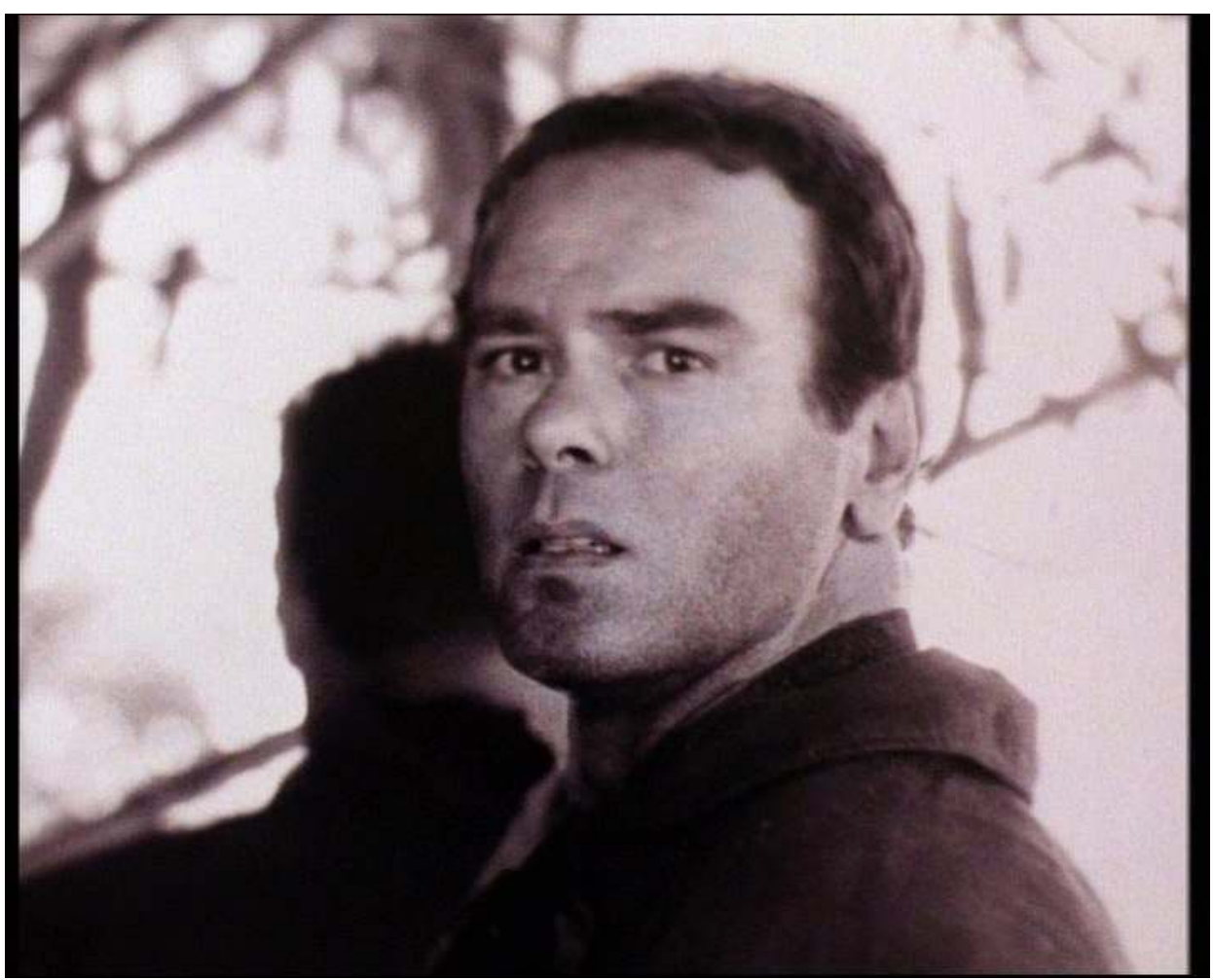

Dans les autres épisodes, les conflits ont toujours lieu hors champ et apparaissent sous forme de récits ou de traces. Les nombreux anciens combattants qui croisent le chemin de Sam Beckett essaient de dire leur expérience et leur traumatisme, parce qu'ils le ressassent. C'est le point nodal de leur existence sur lequel ils reviennent constamment. Ainsi du motard qui revient de la Guerre de Corée dans l'épisode 3.9, «Rebel Without A Clue ", mais aussi, dans un autre épisode, du vétéran de la Seconde Guerre mondiale devenu prêtre. C'est aussi bien sûr le cas d'Al dont l'emprisonnement au Vietnam est un sujet récurrent dans la série.

\subsection{La guerre comme trace}

13 Finalement le récit est lié à la troisième modalité d'existence de la guerre dans la série : celle de la trace, qu'elle soit psychologique ou physique. Plusieurs thèmes majeurs liés à la guerre s'y trouvent convoqués, comme l'attente, le deuil, le trauma, la blessure ou le handicap. Les conflits sont avant tout présentés comme des expériences vécues pour divers personnages, que ce soit pour les combattants ( $\mathrm{Al}$ qui revient et découvre que sa femme s'est remarié pensant qu'il était mort; ceux qui reviennent handicapés, ou traumatisés psychologiquement) ou pour leurs proches, les familles qui attendent le retour du combattant, ou qui le croient mort, celles qui ont perdu un fils. Autant de drames et de cicatrices. Le créateur de la série, Donald Bellisario, est lui-même un ancien marine et beaucoup de ses séries présentent des personnages de militaires: ainsi Magnum, est-il un vétéran du Vietnam, ce qui apparaît explicitement dès le premier épisode ${ }^{8}$. 

champ, elle concerne tout un chacun. Ainsi quand, dans "Star-Crossed ", le second épisode de la série, qui se passe en juin 1972, Sam revoit une femme qu'il a aimée dans sa jeunesse et se dit qu'il a peut-être voyagé dans le temps pour changer ce qui s'est passé et qu'ils finissent ensemble, un journal télévisé parle de la guerre. Sam commente en voix off: « There was a time when I cared about politics and the making of history but the big picture seemed unimportant next to getting a chance for Donna and I in the future. »Or, Sam n'est pas là pour lui-même mais pour la rapprocher de son père qu'elle n'a pas vu depuis des années. Un père qui, justement, est sur le point de partir au Vietnam. En réalité, le journal télévisé n'était pas anodin puisqu'il renvoie directement au destin des personnages principaux de l'épisode. Cette histoire que Sam souhaiterait laisser de côté finit par le rattraper et s'installe au cœur des enjeux réels de l'épisode, comme si le personnage ne pouvait pas y échapper, rappelant aussi la dimension altruiste et sacrificielle de la mission de Sam. L'histoire et la guerre ne sont pas une toile de fond mais un enjeu majeur.

\section{L'expérience intime de la guerre}

15 Ces différentes guerres touchent donc l'ensemble de la population, non pas de manière abstraite et générale mais bien appliquée à chaque individu dans son expérience de vie. Ce n'est pas un hasard si c'est le cas des deux personnages principaux, puisque le grand frère de Sam, Tom, meurt au Vietnam (avant que le scientifique ne change cette version de l'histoire), et que par ailleurs, $\mathrm{Al} \mathrm{y} \mathrm{est} \mathrm{resté} \mathrm{prisonnier} \mathrm{plusieurs} \mathrm{années} \mathrm{et} \mathrm{découvre} \mathrm{à}$ son retour que sa femme s'est remariée pensant qu'il était mort. Ce sont des drames originels, certes des topoï des récits de guerre, mais qui hantent constamment les protagonistes et définissent leur parcours.

\subsection{La hantise de la guerre du Vietnam}

C'est le cas aussi des personnages secondaires que Sam doit aider. En cela la guerre fonctionne dans la série comme un traumatisme, au sens où l'emploie Freud dans Au-delà du principe de plaisir ${ }^{9}$, c'est-à-dire un événement que l'on ressasse sans cesse et qu'il est difficile de dépasser. Pour ces personnages qui souffrent parfois de syndrome de stress post-traumatique, la guerre se poursuit dans le difficile retour à la vie civile (comme pour le motard traumatisé par la guerre de Corée), dans la marque indélébile de la blessure (comme pour le vétéran paraplégique qui essaie de se suicider dans l'épisode 5.4) ou l'impossible retour à la vie d'avant, comme c'est le cas pour Al qui a perdu sa femme. Celui-ci déclare ironiquement dans l'épisode 5.4 qui se déroule dans un hôpital de vétérans pendant la guerre du Vietnam: « we thought that the war was over when we left the jungle ». C'est bien là le propos de la série: on n'y voit pas le combattant en tant que combattant, mais avant et après la guerre. Il n'est pas présenté dans un récit épique ou même héroïque mais toujours dans la souffrance. Au mieux, il a survécu. De fait, cette présentation systématiquement pathétique ou tragique renforce le propos pacifiste de la série.

17 C'est également ce qui est mis en scène dans «The Beast Within» (5.19), qui porte sur des anciens combattants du Vietnam vivant, comme Rambo dans First Blood (Ted Kotcheff, 1982), isolés dans la forêt. Al y explique à Sam qu'ils vivent ainsi parce que « s'il n'y a pas d'hommes, il n'y a pas de guerres ». Ces vétérans partagent un traumatisme - la mort d'un 
de leurs amis - dont on va revoir les circonstances dans un flashback : dans cet épisode, l'idée même d'analepse souligne la dimension spectrale du combat dans l'image, puisque la mise en image des événements passés vient hanter le présent d'énonciation.

D'autre part, il ne faut pas oublier aussi que nous sommes dans une série, et plus précisément dans ce que j'appelle une série semi-feuilletonnante formulaire ${ }^{10}$, qui aborde l'histoire de manière non-linéaire. Les sauts de Sam sont aléatoires et il avance et recule dans le temps constamment, ce qui fait que les différentes guerres évoquées, et en particulier celle du Vietnam, qui est la plus présente dans la série, fonctionnent comme un motif récurrent. On y revient toujours, le motif réapparaît sans cesse, avec des variations mais aussi des constantes: la figure de la femme qui rencontre un autre homme pensant que son mari est mort au front par exemple apparait plusieurs fois. Dès lors, la guerre du Vietnam devient un motif qui hante la série, et par conséquent l'histoire américaine.

19 La hantise joue sur la double modalité de l'absence et de la présence, qui sont justement deux thèmes fondamentaux dans le rapport à la guerre. Cet aspect ressort particulièrement dans l'épisode sur la première femme d'Al. Le final de l'épisode est construit entièrement sur ce motif. Al, avant que Sam ne saute dans le temps, va voir une dernière fois sa femme, mais elle ne peut pas le voir, car elle appartient à un autre temps. Cette scène fondamentale, puisqu'elle sera reprise et prolongée dans la toute dernière scène de la série, met en scène la présence/ absence d'Al pour Beth qui répond à la présence/absence de Beth pour lui. Ils cohabitent dans un même espace mais sont séparés irrémédiablement par le temps.

Figure $4:$ Al et sa première femme Beth (5.19)

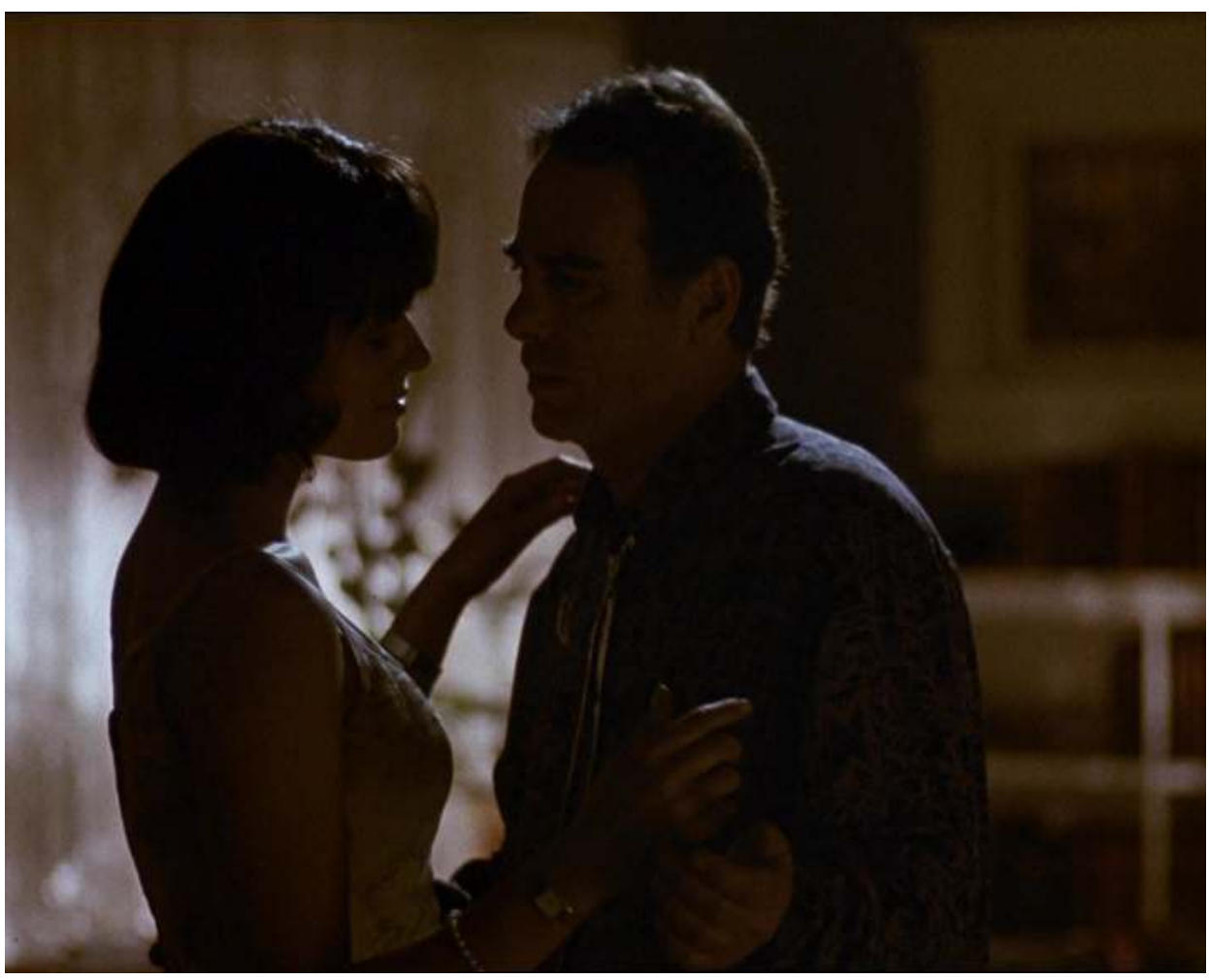

Tous ces éléments soulignent la dimension dramatique, voire tragique de la guerre, qui font ainsi émerger un discours pacifiste. D'ailleurs, Sam ne cesse de dénoncer la guerre 
(notamment la guerre du Vietnam) et son absurdité. Il ne perd jamais une occasion de prendre position sur cette question. Ainsi, dans l'épisode 4.11, "The Play's The Thing", Sam débat avec un entrepreneur dans le domaine du textile dont le principal client est l'armée américaine. Ce dernier se félicite du fait que la demande est très importante pour le Vietnam et Sam lui répond ironiquement : "Vous avez l'air de penser que c'est une bonne chose ${ }^{11} \ldots$.. Sam est alors identifié par un autre personnage comme un militant anti-guerre et l'entrepreneur lui lance: «Ah, vous êtes donc anti-américain ${ }^{12}$.» Sam lui répond: « Pas du tout. C'est plutôt l'inverse. Mais [...] Bien des hommes vont perdre leur vie pour rien. $»^{13} »$. Ici la position de Sam n'est pas radicale dans la mesure où il se sert surtout de ce qu'il sait de l'avenir pour prendre position, mais les débats sur ce sujet sont récurrents dans la série, et portent sur de multiples conflits, Sam prenant toujours position contre la guerre.

\subsection{Un message pacifiste}

21 De la même façon, dans un épisode sur la guerre froide et la crainte de la guerre atomique, Sam parle à un enfant terrifié qui pense que les Soviétiques représentent le mal parce que son père le lui a dit. Il récuse cette idée et lui explique que les « Russes » sont comme eux, des gens normaux. L'épisode dénonce ici la paranoïa et la violence qu'elle peut causer, et travaille sur la représentation de l'ennemi. Le début de l'épisode semble plonger Sam dans un film de science-fiction, figurant l'effet de la panique. Il rappelle l'épisode 3.3 de The Twilight Zone, "The Shelter", qui porte sur le même type de problématique. Cet état de stress et d'angoisse conduit l'enfant, à la fin de l'épisode, à attaquer un voisin croyant qu'il s'agit d'un soldat russe. Le battage médiatique angoissant et les discours incessants de son père sur le sujet, c'est-à-dire la propagande, transforment son point de vue au point de pousser à l'hallucination. Le réel est alors transfiguré. L'image adopte le point de vue de l'enfant et remplace l'espace d'un instant le voisin par l'image d'un soldat. 
Figure 5 : L'enfant croit voir un soldat (4.11)

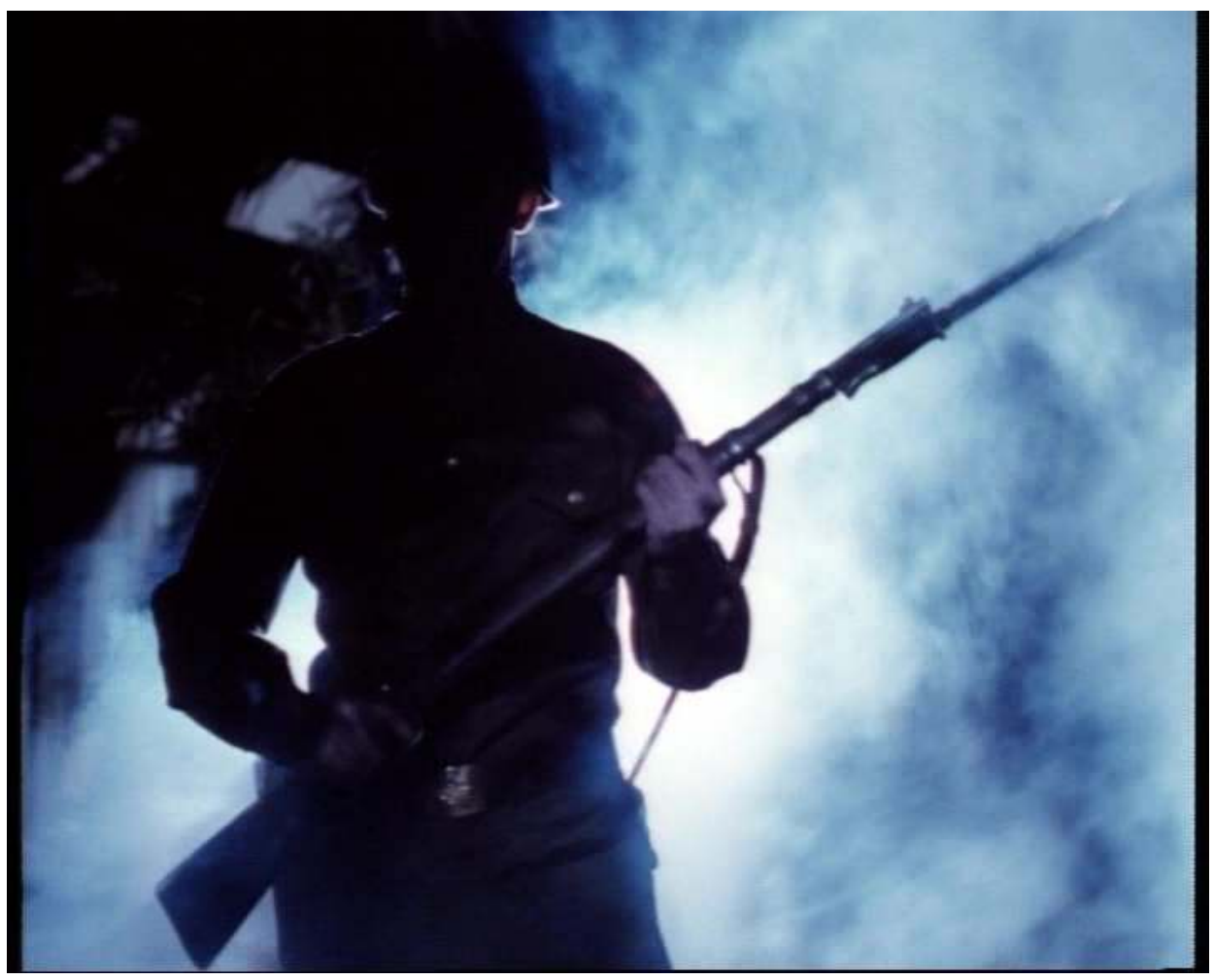

Ces dénonciations de la guerre, de la paranoïa et de la réduction d'un ennemi à une figure du mal, se rapportent bien sûr à des conflits du passé mais résonnent aussi avec le présent de la diffusion de la série, (et même son avenir d'ailleurs) puisqu'elle est diffusée en pleine guerre du Golfe (1990-1991). On sait l'importance que les images télévisées ont eu pour le mouvement pacifiste lors de la guerre du Vietnam, mais aussi l'instrumentalisation de ces mêmes images cette fois au service d'une propagande guerrière lors de la guerre du Golfe. La série s'inscrit dans ce contexte et devient un miroir réflexif de l'usage idéologique de la télévision, en particulier dans la mesure où elle pose régulièrement sa dimension méta-textuelle en travaillant sur les images, au sens large, et leur rapport à l'histoire. Le propos pacifiste y résonne donc avec d'autant plus de force. Comme l'écrit Marjolaine Boutet à propos de la représentation de la guerre du Vietnam dans les séries télévisées :

En ce sens, le discours sur le passé vietnamien est aussi - est surtout - un discours sur le présent. Les représentations de la guerre du Vietnam, en ce qu'elles apportent une justification à cette intervention ou qu'elles la lui refusent, sont révélatrices de la façon dont l'Amérique envisage sa capacité d'intervention dans le monde ${ }^{14}$.

\subsection{Une expérience empathique}

La série repose sur le fait que les sauts dans le temps de Sam sont là pour « réparer des erreurs du passé ». Dès lors, la dimension morale est très importante dans la série, comme le souligne Steve Anderson dans son article "History TV and Cultural Memory " ${ }^{15}$. Sam est le héros par essence, il a toutes les qualités possibles et imaginables : il défend les opprimés, il est empathique et surtout il sacrifie sa propre vie pour aider les autres. En combattant 
l'injustice (racisme, misogynie, homophobie), il la désigne aux spectateurs. Il défend des valeurs. D'ailleurs, il est une sorte d'ange gardien, comme le pitch de la série le souligne puisque Dieu, le temps, ou quelque chose a profité de son expérience scientifique de voyage dans le temps pour l'envoyer en mission. Al le lui dit dans le pilote : "You gotta believe that God or Time or something was just waiting for your quantum leap to correct a mistake. " Mais il ne modifie jamais l'Histoire avec un grand H. Il change la vie d'individus, la petite histoire. Bien sûr, il rencontre des figures historiques, mais son apport est le plus souvent anecdotique : par exemple il apprend à danser le twist à Chubby Checker dans " Good Morning, Peoria » (2.6). Quelque rares cas sont des apports significatifs à l'Histoire : il est ainsi responsable du Watergate, et il sauve Jackie Kennedy. Autrement, il n'intervient pas sur le cours des événements. Il contribue ainsi à la trame de l'histoire en sauvant des victimes collatérales. Évidemment, chacune des actions de Sam sur la vie d'une personne a une série de conséquences positives sur d'autres personnes, notamment leurs proches, mais il s'agit toujours d'une action localisée.

C'est un positionnement crucial parce que, alors même que le contexte politique, social, économique, est toujours très présent, l'histoire telle qu'elle est présentée dans la série est toujours vécue. Le principe même de Quantum Leap, est que Sam expérimente la vie de quelqu'un. La série repose sur l'empathie du personnage mais sa structure elle-même est foncièrement empathique, puisque chaque épisode est construit de manière à prendre conscience de ce que ressent un individu en fonction de son histoire et de ce qu'il a vécu. Cette personne, qui n'était qu'un inconnu pour le personnage et les téléspectateurs au début de l'épisode, devient réellement une personne avec une histoire et des sentiments, une personne à laquelle le protagoniste va s'identifier, permettant ainsi au spectateur de découvrir de l'intérieur son point de vue. Alors même qu'il connaît ces personnes depuis quelques jours seulement, Sam prend soin d'elles, il les aime et il est prêt à tous les sacrifices pour les aider. L'histoire, pour le spectateur, est donc médiée par le personnage de Sam.

Le rapport à la guerre est du même ordre, elle est vécue de l'intérieur par Sam, qui expérimente la souffrance des individus dont la vie a été brisée par ces conflits. De même, l'implication personnelle de Sam et surtout d'Al en tant qu'ancien marine et prisonnier de guerre, va tout à fait dans ce sens. Le but de la série est de faire que nous ne soyions pas spectateurs de l'Histoire, mais que nous la vivions. Même si bien des éléments sont en réalité des réécritures fictionnelles, il est clair que le projet idéologique de la série est celui-là. La récurrence même des motifs au fil des épisodes crée une mémoire émotionnelle des événements pour le spectateur, une sorte de vécu cumulé. Or ce projet est à comprendre dans le contexte de l'importance de la télévision dans les représentations de l'histoire. Comme le souligne Gary Edgerton, «[...] television is the principal means by which most people learn about history today ${ }^{16}$.» Et il ajoute plus loin: « History on TV tends to stress the twin dictates of narrative and biography which ideally expresses television's inveterate tendency towards personalizing all social, cultural, and for our purposes, historical matters within the highly controlled and viewer involving confines of a well-constructed plot structure ${ }^{17}$. »

Cette expérience vécue se cristallise particulièrement dans un des éléments structurants de la série, le saut, et ses effets narratifs. En effet, Sam saute dans le temps et, littéralement, atterrit en plein milieu de l'histoire, au milieu d'événements dont il ne sait rien. L'entrée in medias res, au sein d'une action qu'il ne va pouvoir analyser que dans un deuxième temps, est justement une manière de mettre en valeur l'expérience. Ainsi, dans 
l'épisode où Sam se retrouve amputé des deux jambes parce qu'il est vétéran du Vietnam, il ne sait pas au départ qu'il est amputé et essaie de se lever. Al le prévient alors et il expérimente donc psychologiquement et physiquement ce handicap, dans cette impossibilité soudaine de bouger. Autre exemple, dans l'épisode sur Tom à Saigon, l'arrivée de Sam est comme un écho à l'absurdité de la présence de ces jeunes dans cette guerre. Sam débarque, ne comprenant pas ce qui lui arrive, presque comme ces soldats qui ont été littéralement parachutés dans cette guerre sans forcément l'avoir voulu.

\section{Conclusion : une approche didactique et incarnée de I'Histoire récente des Etats-Unis}

Ces effets créent une prise de conscience du spectateur, dans une dimension didactique évidente pour un programme grand public et familial. L'approche de l'Histoire n'est pas abstraite mais passe par le storytelling, c'est-à-dire le fait de raconter une histoire : tous les éléments évoqués construisent un récit avec des personnages. La série ne nous dit pas que la guerre a fait des milliers de victimes, mais elle nous raconte l'histoire de telle ou telle personne qui a perdu son frère. C'est une stratégie particulièrement efficace pour transmettre un message idéologique.

C'est ainsi que l'histoire dans Quantum Leap peut être à la fois iconique et individualisée. On ne touche jamais à la grande Histoire, on s'attache à la petite mais, en même temps, la Grande Histoire est toujours là abordée à travers un imaginaire culturel qui engage le téléspectateur. Il reconnaît une époque par son style vestimentaire, sa musique, mais aussi se remémore un certain nombre de films ou même de séries télévisées qui dessinent l'ancrage imaginaire de l'épisode. C'est cette double modalité qui construit le discours historique et le commentaire social de la série, notamment en ce qui concerne la guerre.

\section{BIBLIOGRAPHIE}

BOUTET Marjolaine, « Les séries télévisées américaines des années 1980 : une autre histoire de la guerre du Vietnam », Vingtième Siècle, hiver 2004, p. 61-73. (consultable sur https:// www.cairn.info/revue-vingtieme-siecle-revue-d-histoire-2004-4-page-61.htm) BOUTET Marjolaine, « Le Vietnam en prime-time », Le Temps des Médias, avril 2005, p. 188-199. (consultable sur https://www.cairn.info/revue-le-temps-des-medias-2005-1-page-188.htm) BOUTET Marjolaine, "Le Vietnam et l'Amérique à travers le cinéma et la télévision", in Marc Ferro, Pascal Blanchard, Isabelle Veyrat-Masson (dir.), "Guerres et mémoires”, Hermès, n 52, septembre 2008, p. 75-82. (consultable sur https://www.cairn.info/revue-hermes-la-revue-2008-3page-75.htm)

EDGERTON Gary R. et Peter C. Rollins (dir.), Television Histories. Shaping collective Memory in the Media Age, Lexington, The University Press of Kentucky, 2001.

EDGERTON Gary (dir.), Film and History, vol 30.1, Mars 2000. 
FREUD Sigmund, Au-delà du principe de plaisir, [1920] Paris, Payot, 2010.

WIGgins Kayla McKinney, "Epic Heroes, Ethical Issues, And Time Paradoxes In Quantum Leap", in Journal of Popular Film \& Television, vol. 21.3, 1993.

\section{NOTES}

1. Quantum Leap, Donald Bellisario, 1989-1993, NBC, 5 saisons.

2. Cette expression est tirée du discours de présentation de la série qui débute chaque générique : "Theorizing that one could time travel within his own lifetime, Dr. Sam Beckett stepped into the Quantum Leap accelerator and vanished... He woke to find himself trapped in the past, facing mirror images that were not his own and driven by an unknown force to change history for the better. His only guide on this journey is $\mathrm{Al}$, an observer from his own time, who appears in the form of a hologram that only Sam can see and hear. And so Dr. Beckett finds himself leaping from life to life, striving to put right what once went wrong and hoping each time that his next leap will be the leap home."

3. La saison 1 est cependant plus courte (9 épisodes) car Quantum Leap est une série de mid-season (dont la diffusion a commencé le 26 mars 1989).

4. Il n'existe que deux exceptions, les épisodes 4.1 (lorsque Al voyage dans le temps à la place Sam) et 5.20 (où Sam vit un moment de l'existence de son arrière grand-père durant la guerre de Sécession).

5. Cet antépénultième épisode (The Leap Between the States, 5.20) ne respecte pas le principe des voyages de Sam, mais montre l'importance de cette guerre fondatrice pour les scénaristes. Ils font dire à $\mathrm{Al}$ que cette « anomalie " s'explique par la similitude entre l'ADN de Sam et celui de son ancêtre John, capitaine yankee. (http://quantumleap.wikia.com/wiki/ The_Leap_Between_The_States_(episode), consulté le 27 octobre 2016). NDLR.

6. Donald P. Bellisario (né en 1935) a été sergent dans les Marines à la fin des années 1950 avant de se lancer dans une carrière de publicitaire, puis de scénariste pour la télévision. On lui doit notamment les séries Magnum (CBS, 1980-1988), Supercopter (Airwolf, CBS>USA, 1984-1987), JAG (NBC>CBS, 1995-2005) et NCIS (CBS, depuis 2003).

7. La seule autre représentation du champ de bataille est celle de la guerre de Sécession au début de l'épisode 5.20.

8. cf. Marjolaine Boutet, «Les séries télévisées américaines des années 1980 : une autre histoire de la guerre du Vietnam », Vingtième Siècle, hiver 2004, p. 61-73.

9. Freud, Sigmund, Au-delà du principe de plaisir, [1920] Paris, Payot, 2010.

10. Une série semi-feuilletonnante formulaire est une série qui présente des intrigues bouclées construites chaque semaine sur le même modèle et développe cependant une intrigue sur le long terme de la série. J'oppose ce terme aux séries semi-feuilletonnantes épisodiques qui présentent également une tension entre intrigues bouclées et récit au longs cours mais sans suivre un canevas spécifique qui se répète à chaque épisode.

11. "You sound like it's a good thing..."

12. "So you are anti-american."

13. "Not at all. It's quite te opposite. [...] A lot more are going to lose their lives needlessly.»

14. Boutet, Marjolaine, op.cit., II 53. Voir aussi sur ces questions, du même auteur : « Le Vietnam en prime-time", Le Temps des Médias, avril 2005, p. 188-199 et "Le Vietnam et l'Amérique à travers le cinéma et la télévision ", in Marc Ferro, Pascal Blanchard, Isabelle Veyrat-Masson (dir.), “Guerres et mémoires”, Hermès, n 52, septembre 2008, p. 75-82. 
15. Anderson, Steve, « History TV and Cultural Memory », in Edgerton, Gary R., et Rollins, Peter C., éd., Television Histories. Shaping collective Memory in the Media Age, Lexington, The University Press of Kentucky, 2001, p. 31.

16. EDGERTON, Gary , "Television as Historien : an Introduction", in Film and History, vol 30.1, Mars 2000, p. 7.

17. Ibid., p. 8.

\section{RÉSUMÉS}

Cet article analyse la manière dont la série Quantum Leap représente les différents conflits de l'histoire américaine de la seconde moitié du XXe siècle, en particulier la Seconde Guerre mondiale et la guerre du Vietnam. Alors que les champs de bataille sont rarement montrés, la guerre existe partout sous forme de traces, blessures et traumatismes qui hantent l'imaginaire américain. Samuel Beckett visite la grande Histoire par l'intermédiaire des histoires intimes d'individus. La série invite le protagoniste à explorer différents points de vue, différentes expériences de vie, notamment traumatiques, et construit ainsi un propos pacifiste et une dénonciation des souffrances causées par les guerres.

This paper aims at analyzing how Quantum Leap presents the main conflicts in the American history of the second half of the 20th century, particularly World War II and the Vietnam War. Whereas battlefields are seldom shown directly, war exists as traces, wounds and traumas that haunt the American imagery. Samuel Beckett travels through History by living intimate stories. The series pushes the protagonist to explore different points of view and experiences of life, sometimes traumatic, and builds a pacifist discourse and a denunciation of the sufferings caused by wars.

\section{INDEX}

Mots-clés : Code Quantum, guerre, histoire des États-Unis, Seconde Guerre mondiale, guerre du Vietnam, guerre froide, ancien combattant, traumatisme

Keywords : Quantum Leap, war, U.S. history, World War II, Vietnam War, Cold War, veteran, trauma

\section{AUTEUR}

\section{CLAIRE CORNILLON}

Claire Cornillon est PRAG à l'Université Paul Valéry - Montpellier 3. Agrégée de Lettres modernes et certifiée en Anglais, docteure en littérature comparée et membre de GUEST-Normandie, elle est spécialiste de la littérature et du cinéma de science-fiction, des séries télévisées et du transmédia. 